\title{
HIGHLIGHTS
}

\section{Pegvisomant as add-on to somatostatin analogue therapy}

Addition of around $60 \mathrm{mg}$ pegvisomant weekly to somatostatin analogue (SSA) therapy has an equal efficacy as pegvisomant monotherapy, enabling a dose reduction in weekly pegvisomant of more than $50 \%$, according to a new study in the European Journal of Endocrinology.

"Combining somatostatin analogues and pegvisomant decreases endogenous growth hormone (GH) levels," explains lead investigator A. J. Van der Lely (Erasmus MC, Rotterdam). "Also, the liver becomes selectively GH-resistant via several mechanisms. Therefore, the competitive $\mathrm{GH}$ receptor antagonist pegvisomant meets less GH to compete with and needs to block less $\mathrm{GH}$ receptors in the liver in order to decrease insulin-like growth factor 1 (IGF-1) levels."

Van der Lely and co-workers enrolled 92 patients, who were biochemically uncontrolled on SSAs or on pegvisomant monotherapy. All study participants entered a 4-month run-in period, in which they received a monthly $120 \mathrm{mg}$ dose of the SSA lanreotide. Patients biochemically uncontrolled after this period $(n=57)$ entered a 28 -week coadministration period, receiving $120 \mathrm{mg}$ lanreotide per month plus pegvisomant (60 mg once weekly, adapted every 8 weeks based on IGF-1 levels to $40-80 \mathrm{mg}$ once weekly or $40 \mathrm{mg}$ or $60 \mathrm{mg}$ twice weekly). This regimen enabled assessment of the dose at the final visit, as well as the percentage of patients who exhibited normalization of IGF-1 levels at least once during the study period.

Around $80 \%$ of patients had normalized IGF-1 levels on at least one occasion. Although no head-to-head comparison was performed, the researchers confirmed that a dose reduction of more than $50 \%$ in weekly pegvisomant can be expected after a switch from monotherapy to combination therapy.

"As SSAs are more effective in controlling tumor size, the combination might be more attractive for patients with large tumors,"

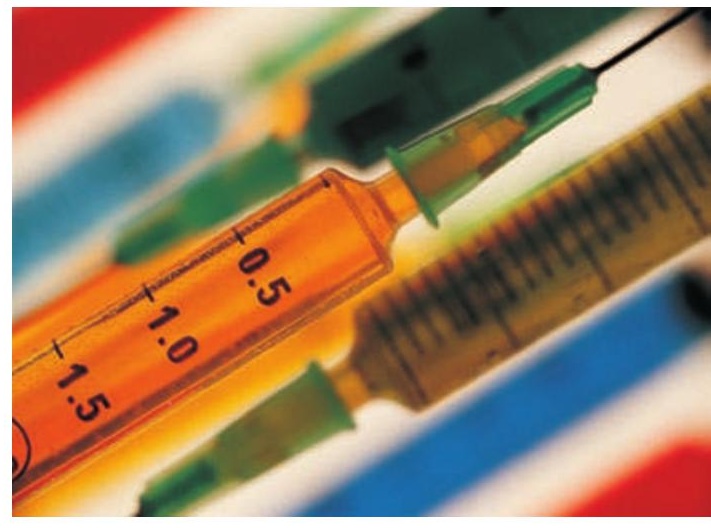

adds Van der Lely. "Also, for patients who need high-dose pegvisomant monotherapy, a dose reduction of say $50 \%$, for example, from $200 \mathrm{mg}$ to $100 \mathrm{mg}$ per week, would save $€ 40,000$ per year." As the mean market price of high-dose SSA monotherapy is around $€ 20,000$ per year in the EU, this reduction would notably reduce costs.

Linda Koch

Original article Van der Lely, A. J. et al. Coadministration of lanreotide Autogel ${ }^{\circledR}$ and pegvisomant normalizes IGF1 levels and is well tolerated in patients with acromegaly partially controlled by somatostatin analogs alone. Eur. J. Endocrinol. doi:10.1530/EJE-10-0867 